\title{
APROXIMACIÓN AL ESTADO DEL ARTE DE LAS ZOMAC EN COLOMBIA ${ }^{1}$
}

\author{
An approach to the state of the art of ZOMAC in Colombia
}

\author{
Edel Rocío Lasso Silva² \\ Institución Universitaria Politécnico Grancolombiano \\ Sonia Patricia Rojas Álvarez ${ }^{3}$ \\ Institución Universitaria Politécnico Grancolombiano \\ Duván Fernando Pinzón Sierra ${ }^{4}$ \\ Institución Universitaria Politécnico Grancolombiano
}

\begin{abstract}
Resumen
La reforma tributaria contenida en la Ley 1819 de 2016 y su decreto reglamentario 1650 de 2017, fueron relevantes en Colombia para la estructuración de un esquema fiscal con perspectiva del conflicto armado- toda vez que se definieron las Zonas más Afectadas por el Conflicto Armado (ZOMAC), como estrategia para reactivar las regiones que han sufrido las acciones negativas de los grupos armados al margen de la ley. El proceso de acuerdo de paz en Colombia busca la construcción de la paz territorial y ambiental, estimulando la creación de nuevas empresas o negocios, las ZOMAC, generando incentivos de tipo tributario específicamente en el impuesto sobre la renta y complementarios, por el hecho de iniciar actividades en estos municipios.

Esta investigación busca comprender los aspectos sociales y tributarios durante los años 2017 y 2018 en las ZOMAC. La investigación presentada es de tipo documental y se propone como un artículo de revisión. Se construyeron las categorías necesarias para el análisis e identificación de los trabajos efectuados en torno a las ZOMAC. De los 27 trabajos de investigación analizados se destaca, en primer lugar, que estos corresponden en un $59,26 \%$ a investigación formativa, corroborando el interés en estudiantes de pregrado, especialmente de la disciplina de contaduría; en segundo lugar, los conceptos con mayor importancia son: impuestos, renta, conflicto armado, posacuerdo, posconflicto, los sectores rurales que desarrollan actividades agrícolas y agropecuarias y aspectos sociales. Por último, la metodología que se presentó en las investigaciones documentadas corresponde en un $80 \%$ de los trabajos al enfoque cualitativo y de tipo descriptivo.
\end{abstract}

Palabras clave: Acuerdo de paz, Conflicto, Impuestos, ZOMAC

\footnotetext{
${ }^{1}$ Citar este artículo como: Lasso, E.R., Rojas, S., Pinzón, D. (2020). Aproximación al estado del arte de las ZOMAC en Colombia, Revista Punto de Vista, 12 (17), pp. 49-62.

2 Docente Politécnico Grancolombiano, Bogotá (Colombia), erlassos@poligran.edu.co. Vinculada al grupo de investigación EICOS- Equipo de Investigación en Contabilidad, Organizaciones y Sociedad.

3 Docente Politécnico Grancolombiano, Bogotá (Colombia), sprojasa@poligran.edu.co. Vinculada al grupo de investigación EICOS- Equipo de Investigación en Contabilidad, Organizaciones y Sociedad.

4 Estudiante e Integrante del Semillero Estudios Fiscales y Contables Politécnico Grancolombiano, Bogotá (Colombia), dupinzon@poligran.edu.co.
}

REVISTA PUNTO DE VISTA VOL. I2 No. 17 (2020) 


\begin{abstract}
The tax reform Law 1819 of 2016 and its regulatory decree 1650 of 2017, were relevant in Colombia for the structuring of a fiscal scheme with an armed conflict perspective. The Zones Most Affected by the Armed Conflict (ZOMAC) were defined, such as strategy to reactivate regions that have suffered negative actions from illegal armed groups. The peace agreement process in Colombia seeks the construction of territorial and environmental peace, stimulating the creation of new companies or ZOMAC businesses, generating tax-type incentives specifically in the income tax and complementary, for starting economic activities in these municipalities. This research seeks to understand the social and tax aspects that have been carried out during the years 2017 and 2018 in the ZOMAC. The research presented is of a documentary type, which provided the elements for the construction of categories necessary for the analysis and identification of the work carried out around the ZOMAC. Of the 27 research papers analyzed, it stands out, firstly, that $59.26 \%$ correspond to formative research, corroborating the interest in undergraduate students, especially in the accounting discipline; secondly, the most important concepts are: taxes, income, armed conflict, post-agreement, post-conflict, rural sectors that carry out agricultural and livestock activities and social aspects. Finally, the methodology that was presented in the documented investigations corresponds in $80 \%$ of the studies to the qualitative and descriptive approach.
\end{abstract}

Keywords: Peace agreement, Conflict, taxes, ZOMAC

\title{
INTRODUCCIÓN
}

Las zonas más afectadas por el conflicto armado (ZOMAC) tienen su origen, en parte, en el Acuerdo Paz firmado en el año 2016 entre el gobierno de Juan Manuel Santos y la guerrilla de las Fuerzas Armadas Revolucionarias de Colombia -FARC. Para entonces, el gobierno nacional delegó al Ministerio de Hacienda y Crédito Público, al Departamento Nacional de Planeación y a la Agencia de Renovación del Territorio, quienes realizaron las proyecciones requeridas, principalmente para el contexto de la deuda social del Estado colombiano, llevando a cabo la reactivación de las ZOMAC. En este escenario surgió la reforma tributaria del año 2016, mediante la cual el gobierno colombiano definió beneficios y descuentos tributarios a las personas jurídicas que quieran voluntariamente desarrollar sus actividades o proyectos en estas zonas, esto con el fin de fomentar el desarrollo en regiones que han sido golpeadas por los conflictos armados del país, y así contribuir con la generación de empleo y el desarrollo industrial. La estrategia utilizada para lograr este propósito se basa en estimular a los empresarios a constituir sus empresas o realizar proyectos en los 344 municipios que se establecieron en el decreto 1650 del 09 de octubre de 2017, brindándoles alivios tributarios y beneficios que solo se dan en estos municipios. La duración de los beneficios e incentivos tributarios en el marco de la Ley 1819 de 2016 es de 10 años; de tal forma, que su progresividad en el impuesto sobre la renta y complementarios se iguale a las leyes tributarias de régimen ordinario, ya que estas ZOMAC están bajo el enfoque de un régimen tributario especial.

En Colombia los impuestos se definen según el artículo 95 numeral 9 de la Constitución Política de Colombia (1991) con el propósito de: "Contribuir al financiamiento de los gastos e inversiones del Estado dentro de conceptos de justicia y equidad". Adicional a ello el Congreso crea las leyes del sistema tributario según el artículo 150, aun así las Asambleas, los Concejos Distritales y Municipios pueden crear sus propios tributos, conllevando a una diversidad de impuestos en el país como lo son: el impuesto a las ventas, renta y complementarios, impuesto nacional al consumo, riqueza entre otros, con estos impuestos se busca proveer dinero al Estado para cubrir el gasto público, inversión y financiación que se requiere para obras y sostenimiento de las necesidades básicas. 
Las zonas más afectadas por el conflicto armado (ZOMAC), son un conjunto de municipios que han sufrido por las acciones de los grupos armados en Colombia al margen de la ley; estas se definieron en el numeral 6 del artículo 236 de la ley 1819 del 29 de diciembre de 2016, la cual estableció 344 municipios que conforman las ZOMAC, las cuales se estipularon en el anexo 2 del decreto 1650 del o9 de octubre de 2017. De acuerdo con el marco normativo referenciado, estos municipios, para denominarse ZOMAC presentan las siguientes características: a) pobreza multidimensional ${ }^{5}$ mayor al $49 \%$, b) debilidad institucional ${ }^{6}$, c) que no sean capaces de generar sus propios ingresos, d) e en los últimos 10 años el conflicto ha sido especialmente intenso y e) no están muy cerca de las capitales (Ver figura 1).

\begin{tabular}{|c|c|}
\hline DEPARTAMENTO & MUNICIPIOS \\
\hline ANTIOQUIA & 55 \\
\hline ARAUCA & 7 \\
\hline BOLIVAR & 16 \\
\hline BOYACA & 5 \\
\hline CALDAS & 9 \\
\hline CAQUETA & 16 \\
\hline CASANARE & 14 \\
\hline CAUCA & 23 \\
\hline CESAR & 15 \\
\hline CHOCO & 18 \\
\hline CORDOBA & 5 \\
\hline CUNDINAMARCA & 12 \\
\hline GUAVIARE & 4 \\
\hline HUILA & 7 \\
\hline
\end{tabular}

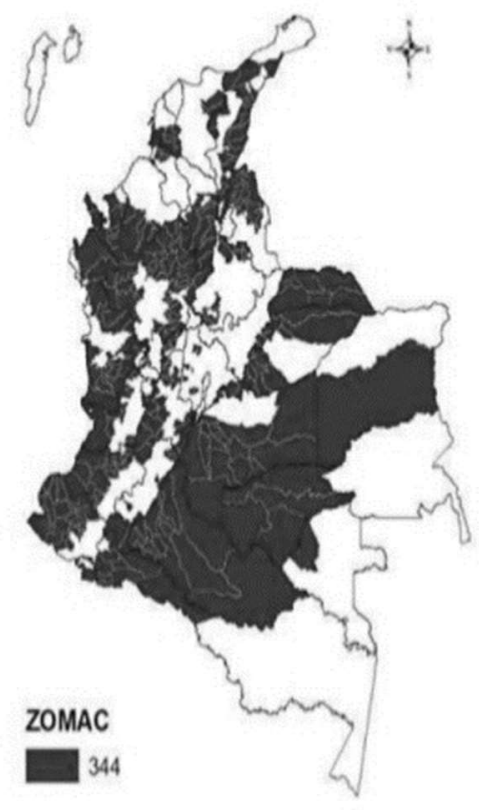

\begin{tabular}{|c|c|}
\hline DEPARTAMENTO & MUNICIPIOS \\
\hline GUAJIRA & 9 \\
\hline MAGDALENA & 2 \\
\hline META & 20 \\
\hline NARINO & 23 \\
\hline N. SANTANDER & 12 \\
\hline PUTUMAYO & 9 \\
\hline QUINDIO & 3 \\
\hline RISARALDA & 4 \\
\hline SANTANDER & 10 \\
\hline CHOCO & 18 \\
\hline SUCRE & 8 \\
\hline TOLIMA & 21 \\
\hline VALLE DEL CAUCA & 15 \\
\hline VAUPÉS & 1 \\
\hline VICHADA & 1 \\
\hline
\end{tabular}

Figura 1. Relación de beneficiarios por cantidad de municipios en cada departamento. Fuente: Colombia Renace Posconflicto

El decreto 1650 de 2017 indica que las nuevas sociedades micro, pequeñas y grandes empresas, que tengan el domicilio principal y desarrollen todas sus actividades en la ZOMAC deberán cumplir con los requisitos de montos mínimos de inversión y generación de empleo directo, para contar con el incentivo tributario respectivo; teniendo en cuenta los siguientes aspectos:

- Sus productos, servicios y comercio se realizan dentro y desde las zonas más afectadas por el conflicto armado ZOMAC. Ios productos o servicios resultantes de estas actividades podrán ser ofrecidos dentro o hacia otras partes del país o del exterior.

- Debe generar empleo directo a las personas a través de contratos laborales desde el 29 de diciembre del 2016 hasta el 31 de diciembre de 2027.

- Se establece un monto mínimo de propiedad, planta y equipo e inventario utilizado en las actividades generadoras de renta, según el tipo de empresa y sus activos totales. Según el tipo de empresa así: microempresa - activos totales hasta 501 SMMLV; pequeña empresa - activos totales entre 502 y 5.001

\footnotetext{
5 El Programa de las Naciones Unidas para el Desarrollo (PNUD), 2015; la define como un concepto más allá de la falta de ingresos para satisfacer necesidades, donde se considera como las personas sufren carencias en las dimensiones: educación, salud, trabajo, seguridad social, vivienda y nivel de vida en general.

${ }^{6}$ Renata Terrazas de FUNDAR, Centro de análisis e investigación, México, 2017; la traduce como la incapacidad del Estado de implementar sus leyes, de combatir el narco y el crimen organizado, de recolectar impuestos, de asegurar los derechos de su población y de mantener las condiciones mínimas para una vida digna.
} 
SMMLV; mediana empresa - activos totales entre 5.002 y 15.000 SMMLV y grande empresa - activos totales desde 15.001 SMMLV.

- El cambio de razón social al cual adiciona la expresión "ZOMAC" al final del nombre.

Adicionalmente, son beneficiaros de esto, aquellos que cumplan con los requisitos establecidos por el decreto 1650 del 09 de octubre de 2017, el cual dicta que aquellos contribuyentes deben estar constituidos e inscritos en la correspondiente Cámara de Comercio de la jurisdicción del municipio en el cual se generara la actividad.

Las obligaciones del contribuyente del régimen tributario de las nuevas sociedades ZOMAC, establece que deben cumplir con la inscripción y actualización del Registro Único Tributario (RUT), indicar la condición de micro, mediana o grande empresa según corresponda, la certificación anual del contador o revisor fiscal y reportar a la Dirección de Impuestos y Aduanas Nacionales (DIAN) la información exógena.

Adicionalmente, la retención en la fuente y autorretención a título de impuesto sobre la renta, cuando se realicen transacciones a los beneficiarios del incentivo tributario se manejará con la tarifa de retención en la fuente en forma proporcional al porcentaje de la tarifa impuesta sobre la renta y complementarios de la sociedad beneficiaria así:

1. Del 2017 al 2021 (6 años), micro y pequeñas empresas el $0 \%$ y medianas y grandes empresas el $50 \%$ de la tarifa general del impuesto de renta.

2. Del 2020 al 2024 (3 años), micro y pequeñas empresas el $25 \%$ y medianas y grandes empresas el $75 \%$ de la tarifa general del impuesto de renta.

3. Del 2025 al 2027 (3 años), micro y pequeñas empresas el $50 \%$ y medianas y grandes empresas el $75 \%$ de la tarifa general del impuesto de renta.

El régimen especial lo perderán las empresas constituidas en la ZOMAC cuando: se cambia de domicilio principal a una zona que no se haya declarado como ZOMAC, se desarrollen la actividad económica fuera del territorio ZOMAC, se incumplan con los requisitos de inversión y empleo presentes en el decreto mencionado anteriormente. También se perderá los beneficios tributarios para el año gravable en el cual se haga el retiro del régimen y se calculará el impuesto sobre la tarifa general establecida en el Estatuto tributario o la tarifa de ganancia ocasional según el caso.

El sentido social del proyecto de las ZOMAC consiste en disminuir por 10 años las obligaciones tributarias, a través de beneficios e incentivos para las empresas constituidas en esos lugares. Adicionalmente, generar empleo, inversión, crecimiento y desarrollo de las regiones que han sido afectadas por el conflicto armado durante ocho décadas; de esta forma, se pretende la igualdad y bienestar social para sus habitantes.

Un aspecto para resaltar, son los requisitos que el legislador estableció para la generación de empleo en las ZOMAC, tomando como base los salarios mínimos y el número de trabajadores dependiendo del tipo de empresa, al igual que fijó los sectores para aplicar dichos beneficios: agricultura, ganadería, caza, explotación de minerales y actividades de servicios conexas así:

- Microempresas: una inversión de 40 SMLMV y dos empleados.

- Pequeña empresa: una inversión de 343 SMLMV y 3 empleados.

- Mediana: una inversión de 1.453 SMLMV y 10 empleados.

- Grandes: una inversión de 7.800 SMLMV y 49 empleados.

De esta forma, también se establecieron los criterios de inversión para 95 divisiones económicas, según el decreto 1650 de 2017. Uno de los sectores con mayor representación en las ZOMAC es el sector palmero que cobija la 
inversión en empresas nuevas que se dediquen al procesamiento y transformación de la palma, así como plantas extractoras, refinadoras, biorrefinerias e industrias de alimentos.

Igualmente, el decreto 1650 de 2017 contempla las obras por impuestos (OxI), las cuales permiten que el contribuyente invierta parte del pago del impuesto sobre la renta, al desarrollo de una obra de infraestructura en cualquiera de las ZOMAC, y con esto apoyar al progreso del municipio y departamento para alcanzar los planes de desarrollo territoriales. Esto hace posible que las empresas mejoren su competitividad con bienes públicos de infraestructura, pues son ellas mismas las que directamente transforman en un 50\% del pago de impuesto en la obra de interés que también debe beneficiar a la comunidad, es decir el 50\% de impuesto que tengan para pagar será destinado al pago de la obra y el otro 50\% será destinado a la Dirección de Impuestos y Adunas Nacionales (DIAN). Los proyectos que se pueden pagar con esta opción son los sectores de: agua potable o alcantarillado, energía, salud pública, educación pública e infraestructura vial.

Cabe señalar, que el objetivo de la investigación es hacer un balance de los aspectos sociales y tributarios que se de las ZOMAC entre 2017 y 2018; como punto de partida, se realizó la construcción del estado del arte con el fin de obtener los elementos necesarios de análisis e identificación de los trabajos académicos, resultados de investigación y artículos de reflexión efectuados en torno a las ZOMAC.

\section{MÉTODO}

La exploración que se presenta es de carácter cualitativo, dado que el interés es interpretar y comprender lo que expresan los textos relacionados con el tema de la ZOMAC, específicamente los aspectos sociales y tributarios. Por tanto, la investigación de tipo documental aporta elementos para la reconstrucción del conocimiento a partir de la lectura y análisis de documentos escritos producidos con anterioridad de forma sistematizada (Gómez, 2011). Desde este enfoque se realizó el estado del arte de las ZOMAC en Colombia, tomando como criterios; la descripción de los componentes teóricos, luego se estableció las siguientes categorías: las regiones afectadas por el conflicto armado, impuestos vinculados a estas y los efectos sociales, considerando como cobertura de tiempo entre los años 2016 al 2020. Una vez definida las categorías se obtuvo la unidad de análisis.

Adicionalmente, se realizó la limitación de las categorías de los temas así: ZOMAC en general, aspectos tributarios y diferentes áreas del país que tienen un efecto social, seguido de la búsqueda de significados para el desarrollo teórico abordado por los autores, las fuentes y los contextos. Para determinar los trabajos se efectúo una revisión sistemática en las bases de datos como: Google académico, Scielo, Dialnet y Redalyc, asimismo se diseñó una matriz diagnosis que incluyo la fuente, tipo de trabajo, título, fecha, resumen, palabras claves, nacionalidad, autores, metodología y bibliografía relevante, lo cual permitió el análisis e interpretación de las investigaciones realizadas durante los años 2016 hasta 2020. Para la recolección de datos se elaboraron fichas de resumen analítico de estudio (RAE) que reunió los conceptos dados por los autores, los cuales aportaron información relevante para el análisis, comprensión e interpretación de los componentes teóricos que se tratan en torno a las zonas más afectadas por el conflicto armado en Colombia.

Por último, el desarrollo del estado del arte configuró las siguientes fases:

- Definición del propósito de la reconstrucción del nuevo conocimiento sobre las ZOMAC.

- Revisión de qué se ha escrito y publicado del área propuesta en bases de datos académicas.

- Formulación rigurosa del procedimiento, de forma ordenada y lógica para el análisis crítico de la información relevante de los contenidos en los documentos escritos y publicados según las categorías definidas.

- Recolección y sistematización de los datos obtenidos como proceso deductivo.

- Interpretación y nueva construcción del conocimiento del tema abordado, de manera metodológica y epistemológica. 


\section{RESULTADOS}

Las investigaciones categorizadas en torno de las ZOMAC se tomaron de 27 trabajos de investigación a partir del año 2016, considerando la creación de estas en Colombia, donde los temas representativos corresponden a: el $37,03 \%$ al sector agropecuario y agrícola y zonas rurales en Colombia; el 14,81\% aborda sobre posconflicto, paz, conflicto, posacuerdo, guerra, responsabilidad social y empresarial, población vulnerable, inseguridad, violencia y delitos, y el 48,14\% impuestos, reforma tributaria, gasto público, economía, planeación y beneficios e incentivos tributarios.

De igual forma, se analizó el método y tipo de investigación, a este respecto se encontró que: ocho (8) son de enfoque cualitativo y tipo descriptivo representando el 29,63\%; uno (1) corresponde a cualitativo y comparativo; uno (1) cualitativo y tipo descriptivo-exploratorio y uno (1) cualitativo y tipo estudio de caso, uno (1) cualitativo y tipo inductivo-descriptivo: uno (1) Mixto, estudio de caso; representando cada uno el 3,70\% del total; dos (2) cualitativo exploratorio, siendo el 7,41\%; cinco (5) cuantitativo, descriptivo correspondiente al $18,52 \%$; tres (3) mixto, descriptivo siendo el $11,11 \%$ y cuatro (4) que representan el $14,81 \%$ no identifican metodología alguna.

A continuación, se describe los trabajos abordados tomando en cuenta el tema objeto de estudio del proyecto de investigación para identificar el contexto así:

\begin{tabular}{|c|c|c|c|c|}
\hline Año & Título & Tipo de trabajo & Metodología & Elementos conceptuales \\
\hline 2016 & $\begin{array}{l}\text { Conflicto armado y configuración } \\
\text { territorial: elementos para la } \\
\text { consolidación de la paz en } \\
\text { Colombia. (Salazar, 2016) }\end{array}$ & Artículo de reflexión & No se identifica & $\begin{array}{l}\text { Colombia, Conflicto Armado, } \\
\text { Configuración Territorial, } \\
\text { pos-negociación de paz, Paz } \\
\text { Territorial. }\end{array}$ \\
\hline 2018 & $\begin{array}{l}\text { ZIDRES y ZOMAC: socialización de } \\
\text { mecanismos y avances. (Castro, } \\
\text { 2018) }\end{array}$ & $\begin{array}{l}\text { Artículo resultado } \\
\text { de investigación }\end{array}$ & No se identifica & $\begin{array}{l}\text { Régimen especial de renta, } \\
\text { obras por impuesto }\end{array}$ \\
\hline 2019 & $\begin{array}{l}\text { Estudio de localización para una } \\
\text { fábrica dedicada a la producción, } \\
\text { distribución de textiles y } \\
\text { confecciones. (Joven, 2019) }\end{array}$ & $\begin{array}{l}\text { Trabajo de grado de } \\
\text { pregrado de } \\
\text { Ingeniería Industrial }\end{array}$ & $\begin{array}{l}\text { Enfoque de } \\
\text { investigación } \\
\text { cuantitativo de } \\
\text { tipo } \\
\text { descriptivo }\end{array}$ & $\begin{array}{l}\text { Industria textil, producción, } \\
\text { método análisis jerárquico, } \\
\text { localización. }\end{array}$ \\
\hline 2019 & $\begin{array}{l}\text { Estudio de Prefactibilidad para la } \\
\text { creación de una empresa dedicada } \\
\text { al cultivo y comercialización de } \\
\text { Cannabis Medicinal en Yarumal - } \\
\text { Antioquia (Torres \& Gómez, 2019) }\end{array}$ & $\begin{array}{l}\text { Trabajo de grado - } \\
\text { Especialización en } \\
\text { Preparación y } \\
\text { Evaluación de } \\
\text { Proyectos Privados }\end{array}$ & $\begin{array}{l}\text { Enfoque de } \\
\text { investigación } \\
\text { cuantitativo de } \\
\text { tipo } \\
\text { descriptivo }\end{array}$ & $\begin{array}{l}\text { Plantas medicinales, Cultivos } \\
\text { comerciales, Cultivos } \\
\text { industriales, Sector agrícola, } \\
\text { e industrial. }\end{array}$ \\
\hline 2019 & $\begin{array}{l}\text { Análisis de experiencias de modelos } \\
\text { asociativos como mecanismo para } \\
\text { el desarrollo empresarial en la } \\
\text { ruralidad (Steiner \& Ramírez, 2019) }\end{array}$ & $\begin{array}{l}\text { Informe } \\
\text { investigación }\end{array}$ & $\begin{array}{l}\text { Estudio de } \\
\text { caso, Análisis } \\
\text { de modelo } \\
\text { asociativo }\end{array}$ & $\begin{array}{l}\text { Alianzas Productivas, Sector } \\
\text { agropecuario y agrícola, } \\
\text { Productividad, Desarrollo } \\
\text { Empresarial Rural, Modelos } \\
\text { Asociativos, Crédito Agrícola, } \\
\text { Informalidad Laboral }\end{array}$ \\
\hline 2019 & $\begin{array}{l}\text { Rediseño de la cadena de suministro } \\
\text { Hortofrutícola en una zona de } \\
\text { postconflicto colombiano de } \\
\text { acuerdo con los estándares de } \\
\text { distribución logística internacional } \\
\text { (Pinzon.2019) }\end{array}$ & $\begin{array}{l}\text { Trabajo de grado - } \\
\text { Maestría en } \\
\text { Logística Integral. }\end{array}$ & $\begin{array}{l}\text { Tipo } \\
\text { documental } \\
\text { argumentativa } \\
\text { con } \\
\text { alcance } \\
\text { exploratorio }\end{array}$ & 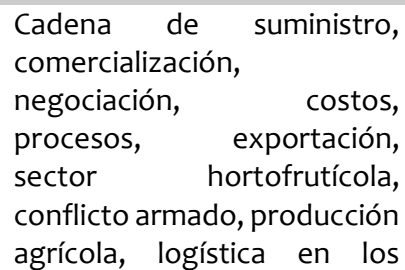 \\
\hline
\end{tabular}




\begin{tabular}{|c|c|c|c|c|}
\hline 2019 & $\begin{array}{l}\text { Financiamiento y calidad del gasto } \\
\text { social (Bonet \& Pérez, 2019) }\end{array}$ & $\begin{array}{l}\text { Trabajo de } \\
\text { investigación - } \\
\text { Centro de Estudios } \\
\text { Económicos } \\
\text { Regionales (CEER) } \\
\text { del Banco de la } \\
\text { República }\end{array}$ & No se identifica & $\begin{array}{l}\text { Financiamiento público local, } \\
\text { calidad del gasto, } \\
\text { proyecciones. }\end{array}$ \\
\hline 2019 & $\begin{array}{l}\text { Seguimiento a la gerencia para } \\
\text { proyectos de vías terciarias en } \\
\text { Colombia valorando alcance, } \\
\text { tiempo y costo, basados en el } \\
\text { PMBOK. (Aranda \& Neira, 2019) }\end{array}$ & $\begin{array}{l}\text { Trabajo de grado - } \\
\text { Especialización de } \\
\text { Gerencia de Obras }\end{array}$ & $\begin{array}{l}\text { Enfoque de } \\
\text { investigación } \\
\text { cualitativo, tipo } \\
\text { descriptivo, } \\
\text { exploratorio }\end{array}$ & $\begin{array}{l}\text { Carreteras, área de } \\
\text { conocimiento, procesos, } \\
\text { gestión del tiempo, gestión } \\
\text { del cronograma, gestión del } \\
\text { alcance. }\end{array}$ \\
\hline 2020 & $\begin{array}{l}\text { Seguimiento y análisis de las } \\
\text { contribuciones de la Ley } 1819 \text { de } \\
\text { 2016. Zonas más afectadas por el } \\
\text { conflicto (ZOMAC), al desarrollo en } \\
\text { caso de estudio en el municipio de } \\
\text { Arauca. (Valencia, 2020) }\end{array}$ & $\begin{array}{l}\text { Trabajo de grado } \\
\text { pregrado de Ciencia } \\
\text { Política }\end{array}$ & $\begin{array}{l}\text { Enfoque de } \\
\text { investigación } \\
\text { cualitativo de } \\
\text { tipo } \\
\text { descriptivo }\end{array}$ & $\begin{array}{l}\text { Posconflicto, } \quad \text { capacidad } \\
\text { institucional, } \\
\text { desarrollo, descentralización }\end{array}$ \\
\hline
\end{tabular}

Tabla 1. Categoría ZOMAC en general. Nota. Elaboración propia.

Los resultados obtenidos de la tabla 1, presenta los temas generales de las ZOMAC realizados en tesis de grado, informes y trabajos de investigación así: cinco (5) trabajos de grado, uno (1) de Ciencia Política, uno (1) de Ingeniería Industrial, dos (2) de Especialización en Preparación y Evaluación de Proyectos Privados y Gerencia de obras y uno (1) de Maestría en Logística Integral. Además, un (1) trabajo de investigación del Centro de Estudios Económicos Regionales (CEER) del Banco de la República, un (1) informe de investigación de Fedesarrollo y dos (2) artículos de reflexión. Cabe señalar, que todas las indagaciones fueron en Colombia.

De los nueve (9) documentos analizados se identificó qué metodología aplicaron, arrojando las siguientes cifras: el $22 \%$ equivalente a dos (2) escritos utilizaron enfoque cualitativo de tipo descriptivo y exploratorio; dos (2) trabajos que corresponde al 22\% desarrollaron la metodología cuantitativo - descriptivo; uno (1) estudio de caso y otro documental representando el $22 \%$ y por último, tres (3) de ellos no identifica método de investigación con una participación del 33\%.

Los conceptos destacados en los trabajos de investigación de la tabla 1 están dirigidos a análisis, estudio de factibilidad, paz, seguimiento de las zonas en posconflicto y conflicto armado; así como, a gerencia, administración, desarrollo rural de las regiones, políticas públicas, cadena de suministro, comercio internacional, actividad agropecuaria, agrícola e industrial, informalidad laboral, obras por impuesto y modelos asociativos en las ZOMAC. 


\begin{tabular}{|c|c|c|c|c|}
\hline Año & Título & Tipo de trabajo & Metodología & Elementos conceptuales \\
\hline 2017 & $\begin{array}{l}\text { Guía de aplicación tributaria para } \\
\text { las pymes familiares (Gamboa \& } \\
\text { Quintero, 2017) }\end{array}$ & $\begin{array}{l}\text { Trabajo de } \\
\text { grado - } \\
\text { Especialización } \\
\text { en Gerencia } \\
\text { Tributaria }\end{array}$ & $\begin{array}{l}\text { Enfoque cualitativo - } \\
\text { Método inductivo, } \\
\text { tipo descriptivo. }\end{array}$ & 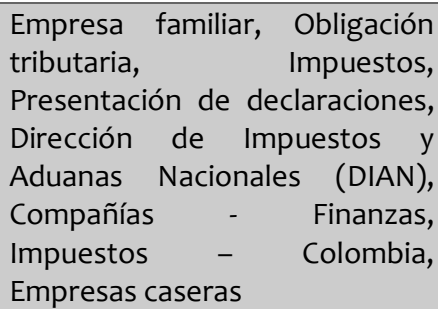 \\
\hline 2018 & $\begin{array}{l}\text { Principales causas tributarias de } \\
\text { quiebre de las pymes en Colombia } \\
\text { (Garcia,2018) }\end{array}$ & $\begin{array}{l}\text { Trabajo de } \\
\text { grado } \\
\text { pregrado } \\
\text { Contaduría } \\
\text { Pública }\end{array}$ & $\begin{array}{l}\text { Enfoque cualitativo - } \\
\text { descriptivo }\end{array}$ & $\begin{array}{l}\text { Beneficios tributarios; } \\
\text { impuestos progresivos y } \\
\text { regresivos; carga tributaria; } \\
\text { fracaso empresarial; planeación } \\
\text { tributaria; pequeña y mediana } \\
\text { empresa; impuestos; incentivos } \\
\text { tributarios. }\end{array}$ \\
\hline 2018 & $\begin{array}{l}\text { Análisis del efecto del IVA en los } \\
\text { hogares de menos ingresos del } \\
\text { departamento de Arauca con los } \\
\text { cambios que ha generado } \\
\text { (Rodríguez \& Mujica,2018) }\end{array}$ & $\begin{array}{l}\text { Trabajo de } \\
\text { grado } \\
\text { pregrado } \\
\text { Contaduría } \\
\text { Pública }\end{array}$ & $\begin{array}{l}\text { Enfoque cualitativo - } \\
\text { descriptivo }\end{array}$ & $\begin{array}{l}\text { Reforma tributaria, Canasta } \\
\text { familiar, Gasto, Impuesto al } \\
\text { valor agregado, Economía. }\end{array}$ \\
\hline 2019 & $\begin{array}{l}\text { Guía didáctica para la apropiación } \\
\text { de los incentivos tributarios del } \\
\text { decreto } 1650 \text { de 2017. ZOMAC } \\
\text { Arauca (Puerto, 2019) }\end{array}$ & $\begin{array}{l}\text { Trabajo de } \\
\text { grado } \quad- \\
\text { Especialización } \\
\text { en Tributaria }\end{array}$ & $\begin{array}{l}\text { Enfoque cuantitativo } \\
\text { de tipo descriptivo no } \\
\text { experimental }\end{array}$ & \begin{tabular}{lrr} 
Tipos de & \multicolumn{2}{c}{ empresas, } \\
características & de le las \\
sociedades, beneficios e \\
incentivos \\
actividades tributarios, \\
$\begin{array}{l}\text { ZOMAC, obligaciones de los } \\
\text { contribuyentes. }\end{array}$
\end{tabular} \\
\hline 2019 & $\begin{array}{l}\text { Incentivo tributario del programa } \\
\text { obras por impuestos - Ley } 1819 \text { de } \\
2016 \text { (Diaz \& Ramos,2019) }\end{array}$ & $\begin{array}{l}\text { Trabajo de } \\
\text { grado } \\
\text { pregrado - } \\
\text { Contaduría } \\
\text { Pública }\end{array}$ & $\begin{array}{l}\text { Enfoque cuantitativo } \\
\text { - descriptivo }\end{array}$ & $\begin{array}{l}\text { Guerra, pobreza, población } \\
\text { vulnerable, políticas públicas, } \\
\text { inseguridad, delincuencia, } \\
\text { conflicto, violencia. }\end{array}$ \\
\hline 2019 & $\begin{array}{l}\text { Análisis de la incidencia fiscal de la } \\
\text { ley } 1819 \text { de } 2016 \text { en una entidad sin } \\
\text { ánimo de lucro (Lance \& } \\
\text { Rubiano,2019) }\end{array}$ & $\begin{array}{l}\text { Trabajo de } \\
\text { grado - } \\
\text { Especialización } \\
\text { en } \\
\text { Administración } \\
\text { y Auditoria } \\
\text { Tributaria }\end{array}$ & $\begin{array}{l}\text { Enfoque cualitativo - } \\
\text { descriptivo }\end{array}$ & $\begin{array}{l}\text { Impuesto de Renta, Beneficio } \\
\text { Tributario, Régimen Tributario } \\
\text { Especial, ESAL, Medidas Fiscales, } \\
\text { Reforma Tributaria, Actividad } \\
\text { Meritoria, Régimen Tributario, } \\
\text { Ordinario, Excedente Fiscal, } \\
\text { Exención Beneficio Neto, } \\
\text { Egresos no Procedentes, Tarifa } \\
\text { Diferencial en Renta }\end{array}$ \\
\hline 2019 & $\begin{array}{l}\text { Obras por Impuestos: Medida para } \\
\text { transformar las zonas más } \\
\text { afectadas por el conflicto armado } \\
\text { en Colombia (Cámara de Comercio, } \\
\text { 2019) }\end{array}$ & $\begin{array}{l}\text { Trabajo de } \\
\text { investigación } \\
\text { entidades } \\
\text { privadas }\end{array}$ & $\begin{array}{l}\text { Enfoque cualitativo y } \\
\text { cuantitativo }\end{array}$ & $\begin{array}{l}\text { Construcción de paz, reforma } \\
\text { tributaria. }\end{array}$ \\
\hline 2019 & $\begin{array}{l}\text { Análisis económico del mecanismo } \\
\text { obras por impuestos (Garizabalo \& } \\
\text { Vargas,2018) }\end{array}$ & $\begin{array}{ll}\text { Tesis } & \text { de } \\
\text { maestría de } \\
\text { Derecho } \\
\text { Económico }\end{array}$ & $\begin{array}{l}\text { Enfoque cualitativo - } \\
\text { descriptivo }\end{array}$ & $\begin{array}{l}\text { Obras por impuestos, Forma de } \\
\text { pago, Extinción de la obligación } \\
\text { tributaria, Impuesto sobre la } \\
\text { renta y complementario. }\end{array}$ \\
\hline 2019 & $\begin{array}{l}\text { Operaciones inafectas y las } \\
\text { exoneraciones fiscales (Marulanda } \\
\text { \& Martinez,2019) }\end{array}$ & $\begin{array}{l}\text { Trabajo grado } \\
\text { pregrado - } \\
\text { Contaduría } \\
\text { Pública }\end{array}$ & $\begin{array}{l}\text { Enfoque cualitativo - } \\
\text { comparativo }\end{array}$ & $\begin{array}{l}\text { Aportes parafiscales, } \\
\text { Exoneración fiscal, Impuesto al } \\
\text { valor agregado IVA, Impuesto } \\
\text { sobre la renta, Operaciones }\end{array}$ \\
\hline
\end{tabular}




\begin{tabular}{|c|c|c|c|c|}
\hline & & & & $\begin{array}{l}\text { inafectas, } \\
\text { tributaria. }\end{array}$ \\
\hline 2019 & $\begin{array}{l}\text { Reflexiones sobre el otorgamiento } \\
\text { de beneficios por parte del Estado } \\
\text { en materia de impuestos. El caso de } \\
\text { la industria avícola (Reflexiones } \\
\text { sobre la concesión de beneficios } \\
\text { otorgados por el Estado en materia } \\
\text { tributaria. El caso de la industria } \\
\text { avícola) (Guerrero,2019) }\end{array}$ & $\begin{array}{l}\text { Artículo de } \\
\text { reflexión }\end{array}$ & No se identifica & $\begin{array}{l}\text { Beneficios fiscales; Impuesto } \\
\text { sobre el valor; Activos exentos. }\end{array}$ \\
\hline
\end{tabular}

Tabla 2. Categoría tributaria. Nota. Elaboración propia

La tabla 2 presenta los resultados de los documentos revisados, analizados e interpretados en torno al tema tributario en las ZOMAC, de lo cual se deduce lo siguiente: en trabajos de grado de especialización, tres (3) de Gerencia Tributaria y Administración y Auditoria Tributaria, en pregrado cuatro (4) del programa de Contaduría Pública, una (1) tesis de maestría de Derecho Económico. Asimismo, un (1) artículo de reflexión y un (1) trabajo de investigación de la Cámara de Comercio.

Las diez (10) investigaciones se realizaron en Colombia y la metodología de investigación que aplicaron en el desarrollo fue: enfoque cualitativo de tipo descriptivo el 40\% correspondiente a cuatro (4) trabajos de grado, cinco (5) trabajos con diferente método así: cualitativo, descriptivo e inductivo, cuantitativo de tipo descriptivo no experimental, cuantitativo - descriptivo, mixto y no define.

Los componentes teóricos de estas investigaciones en torno aspectos tributarios en la ZOMAC han sido: empresas de tipo familiar, pequeña y mediana con sus características societarias, obligaciones de los contribuyentes, impuesto sobre de renta, impuesto al valor agregado e impuesto sobre el valor de activos exentos, beneficios, incentivos y planeación tributaria, temas económicos y sociales tales como: pobreza, población vulnerable, políticas públicas y fiscales, inseguridad, delincuencia, conflicto, violencia, guerra y construcción de paz.

\begin{tabular}{|c|c|c|c|c|}
\hline Año & Título & Tipo de trabajo & Metodología & Elementos conceptuales \\
\hline 2018 & $\begin{array}{l}\text { La corresponsabilidad del } \\
\text { sector privado en la } \\
\text { construcción del posconflicto: } \\
\text { el caso de las ZOMAC (Álvarez, } \\
2018 \text { ) }\end{array}$ & $\begin{array}{l}\text { Artículo de investigación } \\
\text { - Derecho }\end{array}$ & $\begin{array}{l}\text { Enfoque } \\
\text { cualitativo } \\
\text { descriptivo }\end{array}$ & $\begin{array}{l}\text { ZOMAC, violencia, conflicto } \\
\text { - } \text { armado, posconflicto, sector } \\
\text { privado. }\end{array}$ \\
\hline 2018 & $\begin{array}{l}\text { Proyecto socio económico de } \\
\text { transformación de aguacate } \\
\text { Hass por familias del Municipio } \\
\text { de El Tambo Cauca. (Pérez \& } \\
\text { Restrepo, 2018) }\end{array}$ & $\begin{array}{ll}\text { Trabajo grado de } \\
\text { pregrado } & - \text { Ingeniería } \\
\text { Industrial } & \end{array}$ & $\begin{array}{l}\text { Enfoque } \\
\text { cuantitativo } \\
\text { descriptivo }\end{array}$ & $\begin{array}{l}\text { Aguacate Hass, Aguacate, } \\
\text { - Cultivo, Producción. }\end{array}$ \\
\hline 2018 & $\begin{array}{l}\text { Creación de un centro de acopio } \\
\text { lechero Famileche en el } \\
\text { municipio de Herveo - Tolima. } \\
\text { (Londoño \& Corredor, 2018) }\end{array}$ & $\begin{array}{l}\text { Trabajo de grado de } \\
\text { pregrado } \\
\text { Administración } \\
\text { Empresas }\end{array}$ & $\begin{array}{l}\text { Plan } \\
\text { negocio } \\
\text { modelo } \\
\text { Canvas }\end{array}$ & $\begin{array}{l}\text { Económico, social, empleo, plan } \\
\text { de negocio, comercialización, } \\
\text { producción rural. }\end{array}$ \\
\hline 2018 & $\begin{array}{l}\text { Responsabilidad social } \\
\text { empresarial en el proceso de } \\
\text { reincorporación a la vida civil de } \\
\text { los excombatientes de las } \\
\text { FARC-EP (Páez \& Dussan, 2018) }\end{array}$ & $\begin{array}{l}\text { Trabajo de } \\
\text { pregrado } \\
\text { Administración } \\
\text { Empresas }\end{array}$ & $\begin{array}{l}\text { Enfoque } \\
\text { cualitativo } \\
\text { descriptivo }\end{array}$ & $\begin{array}{l}\text { Responsabilidad social, } \\
\text { vocación, integración social y } \\
\text { económica, vida civil, } \\
\text { excombatientes, proceso de } \\
\text { paz. }\end{array}$ \\
\hline
\end{tabular}




\begin{tabular}{|c|c|c|c|c|}
\hline 2018 & $\begin{array}{l}\text { Participación del sector } \\
\text { empresarial en el posconflicto } \\
\text { colombiano (Jiménez, 2018) }\end{array}$ & $\begin{array}{l}\text { Trabajo de grado } \\
\text { pregrado Administración } \\
\text { de Empresas }\end{array}$ & $\begin{array}{l}\text { Estudio } \\
\text { observacional, } \\
\text { analítico, } \\
\text { retrospectivo } \\
\text { y transversal }\end{array}$ & $\begin{array}{l}\text { Posconflicto, Empresa y paz, } \\
\text { Colombia, } \\
\text { responsabilidad } \\
\text { empresarial. }\end{array}$ \\
\hline 2019 & $\begin{array}{l}\text { Incentivos tributarios para el } \\
\text { posacuerdo. ¿una apuesta a la } \\
\text { construcción de paz en } \\
\text { Colombia? (Lasso \& lbáñez, } \\
\text { 2019) }\end{array}$ & Artículo de investigación & $\begin{array}{l}\text { Enfoque } \\
\text { cualitativo } \\
\text { descriptivo }\end{array}$ & $\begin{array}{l}\text { Posacuerdo, Incentivos } \\
\text { tributarios, Construcción de paz, } \\
\text { Colombia }\end{array}$ \\
\hline 2019 & $\begin{array}{l}\text { Crónica de una agonía } \\
\text { anunciada: la crisis del café con } \\
\text { leche (Mora, 2019) }\end{array}$ & Artículo de reflexión & No aplica & $\begin{array}{l}\text { Fertilizantes, cultivos, leche, } \\
\text { café, economía, finanzas. }\end{array}$ \\
\hline 2020 & $\begin{array}{l}\text { Asociatividad empresarial: una } \\
\text { estrategia para las } \\
\text { organizaciones del sector } \\
\text { cacaotero del municipio de } \\
\text { Fortul Arauca (Burgos } \\
\text { \&Fonseca, 2020) }\end{array}$ & Artículo de investigación & $\begin{array}{l}\text { Enfoque } \\
\text { cualitativo } \\
\text { descriptivo }\end{array}$ & $\begin{array}{l}\text { Acción } \\
\text { asociatividad, } \\
\text { diagnóstico. }\end{array}$ \\
\hline
\end{tabular}

Tabla 3. Categoría social. Nota. Elaboración propia

Durante los años 2018 al 2020 los trabajos efectuados en torno al tema de las ZOMAC y que incluye aspectos de índole social, están divididos en la tabla 3 de la siguiente forma: una (1) tesis de pregrado de Ingeniería Industrial y tres (3) de Administración de Empresas. En artículos de investigación dos (2) y uno (1) de reflexión.

Las metodologías que predominan en estas ocho (8) investigaciones se refieren a enfoque cualitativo descriptivo con una participación del 37,5\% y con el 12,5\% están: cuantitativo - descriptivo, plan de negocio modelo canvas y estudio observacional, analítico, retrospectivo y transversal.

El aporte conceptual de las investigaciones realizadas en torno a las ZOMAC en aspectos sociales, están dirigidos a temas tales como: conflicto armado, posconflicto, violencia, actividad económica agropecuaria y agrícola, empleo, comercialización y producción rural, responsabilidad social y empresarial, vocación, integración social y económica, proceso de paz y posacuerdo.

\section{DISCUSIÓN}

El conflicto armado en Colombia permaneció aproximadamente 52 años en auge, lo cual afectó negativamente la paz en el territorio nacional, originando dificultades económicas, sociales y culturales, especialmente en las zonas rurales. De acuerdo con la investigación de Salazar (2016):

la afectación territorial del conflicto armado en Colombia inicia aproximadamente en el año 1986 hasta 2015 de afectación alta y de persistencia en las regiones: Medio y bajo Putumayo, Meta, Caquetá, Villavicencio, Cauca, Norte de Santander, Magdalena, Cesar, Sucre, Bolívar, Antioquia, Choco y Córdoba. Los departamentos de Nariño, Huila, Risaralda y Caldas a partir del año 2012 concentran problemas de violencia. (p. 51)

Un cese parcial al conflicto armado se da una vez la firma del Acuerdo de Paz en el año 2016, concentrando acciones para mitigar el impacto de violencia y gestionar la reactivación económica y social en las zonas afectadas por éste. Surge de este modo, la creación de las zonas más afectadas por el conflicto armado en Colombia 
(ZOMAC) establecidas por las entidades del Estado, conformadas por: el Ministerio de Hacienda y Crédito Público, el Departamento Nacional de Planeación y la Agencia de Renovación del Territorio, quienes definieron los 344 municipios que integran las ZOMAC en función de los siguiente criterios: Índice de desempeño fiscal, municipios priorizados en el programa de desarrollo con enfoque territorial (PDET), índices de pobreza multidimensional, tiempo en vehículo a la capital de departamento, aglomeraciones de sistemas de ciudades, categorías de ruralidad, índices de incidencia de conflicto armado y población, según lo contempla el decreto 1650 de 2017.

En torno a esta nueva acción del estado por gestar una paz territorial y vincular la propuesta de las ZOMAC como un medio de reactivación y confianza en las regiones, las diferentes entidades públicas y privadas en Colombia, inician investigaciones sobre este tema, a partir del año 2016 con la reforma tributaria Ley 1819.

Las temáticas abordadas por los 27 trabajos de investigación presentados en los resultados son:

1. Las regiones afectadas por el conflicto armado, se observó que las investigaciones concentraron sus estudios en: Antioquia, Arauca, Bolívar, Boyacá, Caldas, Caquetá, Casanare, Cauca, Cesar, Chocó, Córdoba, Cundinamarca, Guaviare, Huila, Guajira, Magdalena, Meta, Nariño, Norte Santander, Putumayo, Quindío, Risaralda, Santander, Sucre, Tolima, Valle del Cauca, Vaupés y Vichada. Las regiones analizadas presentan particularidades en cuanto al desarrollo de actividades agrícolas y agropecuarias. En su mayoría, requieren una atención especial para vincular aspectos administrativos, logística, comercialización e infraestructura productiva y vial para consolidar sus proyectos de emprendimiento y crear negocios viables y rentables que puedan dar a conocer sus productos a escala regional y nacional.

2. Impuestos vinculados a estas zonas, por la complejidad de las regiones enmarcadas como las más afectadas por el conflicto armado y la dinámica de las políticas fiscales, el enfoque que predomina en materia de impuestos son: Beneficios e incentivos tributarios, impuestos progresivos y regresivos, carga tributaria, planeación tributaria, Impuesto de Renta y Complementarios, Régimen Tributario Especial, ESAL, medidas fiscales, excedente Fiscal, tarifa Diferencial en Renta, aportes parafiscales, exoneración fiscal e Impuesto al valor agregado IVA. El análisis partió de la interpretación de la reforma tributaria Ley 1819 de 2016 y su decreto reglamentario, con el fin de establecer los requisitos, características y elementos jurídicos que aplican a las empresas constituidas y obras por impuestos en las ZOMAC. Las implicaciones de esta normatividad de carácter especial, configura un desequilibrio empresarial al favorecer las grandes empresas que explotan recursos naturales. Asimismo, no garantiza la sostenibilidad de las micro, pequeñas y medianas empresas después de los diez años del beneficio tributario.

3. Los efectos sociales en las ZOMAC planteados, giran alrededor de la violencia, conflicto armando, posconflicto, sector privado, empleo, fortalecimiento empresarial, actividades agrícolas, ruralidad, responsabilidad social, vocación e integración social y económica, vida civil, excombatientes, proceso y construcción de paz, asociatividad y acción participativa. Los enfoques tratados dirigen su reflexión y análisis en examinar y evaluar las situaciones antes, durante y futuras que implica el Acuerdo de Paz, el cual, no garantiza el cese al fuego, disminución de la violencia, erradicación de los actos ilícitos entre otros para generar prosperidad y bienestar común en estas regiones. Por el contrario, se visualiza aumentó en el desplazamiento forzoso y alto porcentaje del territorio no cultiva lo tradicional de la zona.

Los trabajos de investigación analizados sobre las diferentes temáticas abordadas corresponden en un 59,26\% a investigación formativa, resultado de 16 trabajos de pregrado de áreas: contable, derecho administración de empresas e ingeniería industrial, y el 40,74\% son investigaciones empíricas y aplicadas. Dado que las ZOMAC llevan de creadas aproximadamente cuatro años, los intereses por éstas surgen en la educación superior, especialmente en el pregrado. No obstante, los grupos de investigación han iniciado proyectos dirigidos a comprender e interpretar las implicaciones que trae consigo las ZOMAC, en el contexto jurídico, tributario y social, bajo una 
mirada desde el conflicto armado y el Acuerdo de Paz. Por otro lado, cabe señalar que, en los aspectos metodológicos, prima el enfoque cualitativo de tipo descriptivo.

\section{CONCLUSIONES}

Las 27 investigaciones seleccionadas y revisadas tuvieron en común, estudios dirigidos a las zonas más afectadas por el conflicto armado (ZOMAC) en Colombia. Para el análisis de éstas, se categorizaron los temas de la siguiente forma: ZOMAC en general, aspectos tributarios y sociales, tomando como base trabajos de grado de pregrado y especialización, tesis de maestría, artículos de reflexión y resultado de investigación. Las temáticas que predominaron fueron: acuerdo y construcción de paz, posconflicto, conflicto armado, posacuerdo, administración, desarrollo rural, políticas públicas y fiscales, actividad agropecuaria y agrícola, informalidad laboral, empleo, Impuesto de renta, tipos de empresa, beneficios, incentivos y planeación tributaria, inseguridad, delincuencia, violencia, guerra, responsabilidad social y empresarial, vocación e integración social y económica.

Igualmente, los aspectos metodológicos diseñados en los trabajos de investigación contaron con enfoques cualitativo- descriptivo, cuantitativo-descriptivo, planes de negocio modelo canvas y estudio observacional, destacándose la tendencia por el enfoque cualitativo, por la gama de posibilidades de interpretación que proporciona para la reconstrucción del conocimiento a partir de la mirada subjetiva del investigador.

Es relevante, tener en cuenta que las regiones ZOMAC de los departamentos de: Antioquia, Arauca, Bolívar, Boyacá, Caldas, Caquetá, Casanare, Cauca, Cesar, Choco, Córdoba, Cundinamarca, Guaviare, Huila, Guajira, Magdalena, Meta, Nariño, Norte Santander, Putumayo, Quindío, Risaralda, Santander, Sucre, Tolima, Valle del Cauca, Vaupés y Vichada, han sido por décadas sometidos actos y circunstancias de violencia, guerra, desempleo, desplazamiento, conflictos sociales y económicos que no ha permitido su desarrollo y crecimiento. Por tanto, la intencionalidad de la disposición jurídica y tributaria de la reforma de la ley 1819 de 2016 y sus decretos reglamentarios, es fortalecer y consolidar a partir de la creación de empresas y obras por impuestos la reactivación gradual de estas zonas. De tal forma, que garantice la seguridad, prosperidad y bienestar común. No obstante, el favorecimiento del Estado hacia algunas actividades económicas, y en particular hacia las grandes empresas, dificultará el proceso y construcción de paz territorial, así como la persistencia de cultivos ilícitos.

\section{REFERENCIAS BIBLIOGRÁFICAS}

Álvarez, D. (2018). La corresponsabilidad del sector privado en la construcción del posconflicto: el caso de las ZOMAC. Medellín. Universidad Pontifica Bolivariana.

Aranda, A. \& Neira, W. (2019). Seguimiento a la gerencia para proyectos de vías terciarias en Colombia valorando alcance, tiempo y costo, basados en el PMBOK. Trabajo de Grado. Universidad Católica de Colombia. Facultad de Ingeniería. Programa de Ingeniería de Civil. Especialización en Gerencia de Obras. Bogotá, Colombia.

Bonet, J. Pérez, V. (2019). Financiamiento y calidad del gasto social. Colombia, Bogotá: Banco de la República

Burgos, D. \& Fonseca, D. (2020). Asociatividad empresarial: una estrategia para las organizaciones del sector cacaotero del municipio de Fortul Arauca. Aibi Revista De investigación, administración E ingeniería, 91100. Obtenido de https://doi.org/10.15649/2346030X.621

Cámara de Comercio de Bogotá \& Fundación Ideas para la Paz. (2019) Obras por Impuestos: Medida para transformar las zonas más afectadas por el conflicto armado en Colombia 
Castro, L. E. (2018). ZIDRES y ZOMAC: socialización de mecanismos y avances. Boletín El Palmicultor, (554 Abril), 9-11. Consultado el 11 de julio 2020. https://publicaciones.fedepalma.org/index.php/palmicultor/article/view/12423

Constitución Política de Colombia. Editorial Edijufinancieras Ltda. Bogotá, Colombia, 2001.

Decreto 1650 de 2017. Ministerio de Hacienda y Crédito Público de Colombia, Bogotá, Colombia, 9 de octubre de 2017.

Díaz, D. - Ramos, J. \& Esguerra, F. (2019). Incentivo tributario del programa obras por impuestos -Ley 1819 de 2016. Universidad de la Salle. Consultado el 10 de julio de 2020. https://ciencia.lasalle.edu.co/contaduria_publica/621

Gamboa, C. \& Quintero, A. (2017) Guía de aplicación tributaria para las pymes familiares Universidad La Gran Colombia.

García, A. (2018). Principales causas tributarias de quiebre de las pymes en Colombia. Universidad Militar Nueva Granada. Consultado el 10 de julio de 2020. http://hdl.handle.net/10654/21268.

Garizabalo, L. \& Vargas, M. (2019). Análisis económico del mecanismo obras por impuestos (Trabajo de grado). Pontificia Universidad Javeriana. Bogotá, Colombia.

Gómez, L. (2011). Un espacio para la investigación documental. Revista Vanguardia psicológica clínica teórica y práctica, Vol 1 No.2, marzo 2011. Universidad Manuela Beltrán. Bogotá, Colombia. Consultado el 14 de octubre de 2020. https://dialnet.unirioja.es/servlet/articulo?codigo=4815129

Guerrero, E. (2019). Reflexiones sobre el Otorgamiento de Beneficios por parte del Estado en materia de impuestos. El caso de la industria avícola. Revista Derecho Fiscal N 15, julio-diciembre 2019. Consultado el 12 de julio de 2020. https://ssrn.com/abstract=3470911

Jiménez, O. (2018). Participación del sector empresarial en el posconflicto colombiano. Bogotá. Pontificia Universidad Javeriana

Joven, S.C. (2019). Estudio de localización para una fábrica dedicada a la producción, distribución de textiles y confecciones. Estudio de caso: Grupo Carolina. (Tesis de pregrado en Ingeniería Industrial). Universidad de Ibagué, Tolima, Colombia.

Lance, A. \& Rubiano, C. (2019). Análisis de la incidencia fiscal de la ley 1819 de 2016 en una entidad sin ánimo de lucro. Universidad Jorge Tadeo Lozano.

Lasso, E. \& Ibáñez, J. (2019) Incentivos tributarios para el posacuerdo. ¿Una apuesta a la construcción de paz en Colombia?. V. 4, N. 1, pp. 18-32

Ley 1918 de 2016. Diario Oficial de la República de Colombia No. 50.101, Bogotá, Colombia, 29 de diciembre de 2016.

Londoño, L. \& Corredor, L. (2018). Creación de un centro de acopio lechero Famileche en el municipio de Herveo - Tolima. Corporación Universitaria Minuto de Dios 
Marulanda, A. - Martínez, E. \& González, M. (2017). Operaciones Inafectadas y las exoneraciones fiscales (Tesis de pregrado). Universidad Cooperativa de Colombia, Villavicencio. Consultado el 9 de julio de 2020. http://repository.ucc.edu.co/handle/ucc/10814

Mora, J. (2019) Crónica de una agonía anunciada: la crisis del café con leche. Revista Ideales. Vol. 7, №1

Páez, M. \& Dussan, E. (2018). Responsabilidad social empresarial en el proceso de reincorporación a la vida civil de los excombatientes de las FARC-EP. Bogotá. Universidad del Bosque.

Pérez, J. \& Restrepo, J. (2018) Proyecto Socio Económico de Transformación de Aguacate Hass por familias del Municipio de El Tambo Cauca. Corporación Universitaria Lasallista

Pinzón, A. (2019). Rediseño de la cadena de suministro Hortofrutícola en una zona de postconflicto colombiano de acuerdo con los estándares de distribución logística internacional. Consultado el 8 de julio de 2020. http://hdl.handle.net/10654/32027

Puerto, D. (2019). Guía didáctica para la apropiación de los incentivos tributarios del decreto 1650 de 2017 ZOMAC Arauca (Tesis de especialización). Universidad Libre, San José de Cúcuta - Colombia. Consultado el 9 de julio 2020. https://repository.unilibre.edu.co/bitstream/handle/10901/15532/TRABAJO\%20FINAL\%20ZOMAC.pdf?sequ ence $=1$ \&isAllowed $=y$

Rodríguez, L. \& Mujica, D. (2018). Análisis del efecto del IVA en los hogares de menos ingresos del departamento de Arauca con los cambios que ha generado (Tesis de pregrado). Universidad Cooperativa de Colombia, Arauca. Consultado el 7 de julio de 2020. http://hdl.handle.net/20.500.12494/13424

Salazar, G. S. (2020) El conflicto armado y su incidencia en la configuración territorial: Reflexiones desde la ciencia geográfica para la consolidación de un periodo de pos-negociación del conflicto armado en Colombia, Bitácora Urbano Territorial 2020, (V.26), pp. 45-57. Consultado el 5 de julio de 2020. https://revistas.unal.edu.co/index.php/bitacora/article/view/57605

Steiner, R. \& Ramírez, T. (2019). Análisis de experiencias de modelos asociativos como mecanismo para el desarrollo empresarial en la ruralidad. Bogotá: Fedesarrollo, abril, 85 p.

Torres, A., Gómez. S (2019) Estudio de Prefactibilidad para la creación de una empresa dedicada al cultivo y comercialización de Cannabis Medicinal en Yarumal - Antioquia (Tesis de especialización Preparación y Evaluación de Proyectos Privados) Universidad de Antioquia, Medellín. Colombia.

Valencia, S. (2020). Seguimiento y análisis de las contribuciones de la Ley 1819 de 2016. Zonas más afectadas por el conflicto (ZOMAC), al desarrollo en caso de estudio en el municipio de Arauca.(Tesis de pregrado). Universidad Javeriana de Colombia, Bogotá. Consultado el 9 de julio de 2020. https://repository.javeriana.edu.co/bitstream/handle/10554/50084/Tesis\%20Entrega\%2oFinal\%20ZOMAC\%2 oVEne172020\%20(Javeriana)-converted.pdf?sequence $=1$ 